2000-03-13

\title{
Average Energy Flow of Optical Pulses in Dispersive Media
}

\author{
Scott Glasgow \\ Glasgow@mathematics.byu.edu \\ Michael Ware \\ ware@byu.edu \\ Justin Peatross \\ peat@byu.edu
}

Follow this and additional works at: https://scholarsarchive.byu.edu/facpub

Part of the Astrophysics and Astronomy Commons, and the Physics Commons

Original Publication Citation

Physical Review Letters, vol 84, no 11, pp 237-2373.

\section{BYU ScholarsArchive Citation}

Glasgow, Scott; Ware, Michael; and Peatross, Justin, "Average Energy Flow of Optical Pulses in Dispersive Media" (2000). Faculty Publications. 599.

https://scholarsarchive.byu.edu/facpub/599

This Peer-Reviewed Article is brought to you for free and open access by BYU ScholarsArchive. It has been accepted for inclusion in Faculty Publications by an authorized administrator of BYU ScholarsArchive. For more information, please contact ellen_amatangelo@byu.edu. 


\title{
Average Energy Flow of Optical Pulses in Dispersive Media
}

\author{
J. Peatross, S. A. Glasgow,* and M. Ware \\ Department of Physics and Astronomy, Brigham Young University, Provo, Utah 84602
}

(Received 28 May 1999)

\begin{abstract}
The arrival time of a light pulse at a point in space is defined using a time expectation integral over the Poynting vector. The delay between pulse arrival times at two distinct points is shown to consist of two parts: a spectral superposition of group delays (inverse of group velocity) and a delay due to spectral reshaping via absorption or amplification. The result provides a context wherein group velocity is always meaningful even for broad band pulses and when the group velocity is superluminal or negative. The result imposes luminality on sharply defined pulses.
\end{abstract}

PACS numbers: 42.25.Bs

During the past century some confusion has arisen surrounding the meaning of group velocity in situations where it exceeds the speed of light in vacuum or where it becomes negative. Until recently, the conventional viewpoint has been that in such situations group velocity "has no longer any appreciable physical significance" [1] or "may even lack precise meaning" [2]. In this Letter, we provide a context wherein the function $d k / d \omega$, the inverse of group velocity, always retains significance in connection with the flow of energy in light pulses. The result is a natural consequence of Maxwell's equations, requiring no approximations (e.g., no perturbative expansions about a carrier frequency). It may be applied to pulses of arbitrary bandwidth propagating in a uniform linear medium, even if the spectral width of the pulse encompasses a complicated resonance structure.

In traditional pedagogy, group velocity is often introduced by examining the velocity at which beats propagate when two plane waves of slightly different frequencies interfere $[1,3]$. Group velocity is then defined in the limit as the separation between the two frequencies goes to zero and yields $\partial \omega / \partial k$. Even though this approach necessarily involves more than one frequency, to our knowledge, the importance of group velocity has not previously been demonstrated for light pulses with arbitrarily wide bandwidths.

In 1970, Garrett and McCumber [4] asserted that "the concept of group velocity has meaning for an absorptive medium." Their conclusion was based on simulations of Gaussian pulses propagating in a medium with an absorption resonance centered on the narrow spectrum of the pulse. They found that the peak of a pulse can traverse the medium with apparent superluminal velocities or even exit from the medium before the original peak enters the first surface (i.e., negative velocity). This can occur even while the pulse maintains a semblance of its original temporal profile, albeit attenuated, allowing for an unambiguous assessment of pulse travel time. They explained this seeming affront to the principle of relativity and to causality in terms of pulse reshaping where latter portions of the pulse are preferentially attenuated so that the pulse appears to move forward with excessive speed, which agreed with the group velocity.

In 1981, Chu and Wong [5] confirmed the predictions of Garrett and McCumber by measuring the transmission time of a laser pulse tuned to a resonance in GaP:N. In particular, the physical relevance of group velocity was affirmed in the superluminal and negative cases. More recently, Chiao et al. [6,7] simulated pulse propagation in amplifying media near gain resonances and showed that similar exotic behavior is expected. In all of these works, the refractive index can be represented analytically by a perturbative expansion. Under this narrow band approximation, it is well established that the group velocity plays a clear and prominent role.

The frequency-dependent phase delay associated with propagation through a displacement $\Delta \overrightarrow{\mathrm{r}}$ is often expanded as

$$
\begin{aligned}
\overrightarrow{\mathrm{k}} \cdot \Delta \overrightarrow{\mathrm{r}} \cong & {\left[\left.\overrightarrow{\mathrm{k}}\right|_{\bar{\omega}}+\left.\frac{\partial \overrightarrow{\mathrm{k}}}{\partial \omega}\right|_{\bar{\omega}}(\omega-\bar{\omega})\right.} \\
& \left.+\left.\frac{1}{2} \frac{\partial^{2} \overrightarrow{\mathrm{k}}}{\partial \omega^{2}}\right|_{\bar{\omega}}(\omega-\bar{\omega})^{2}+\ldots\right] \cdot \Delta \overrightarrow{\mathrm{r}} .
\end{aligned}
$$

The coefficient of the linear frequency term is recognized as the inverse of group velocity evaluated at the "carrier" frequency $\bar{\omega}$. Group velocity is often introduced in this manner [2], since it provides a first-order approximation to the speed at which a pulse traverses the displacement $\Delta \overrightarrow{\mathrm{r}}$. However, in the present work, we refer to the function $d \omega / d k$ as group velocity as in Ref. [3], which is permitted to vary arbitrarily within the bandwidth of the pulse. This is critical for dealing with pulses which suffer serious distortion through dispersion. If $\overrightarrow{\mathrm{k}}$ is complex, the imaginary part governs frequency-dependent attenuation (or amplification) and is not used in group velocity.

Within the context of Eq. (1), the significance of the group velocity is limited since it is associated with a single term of the expansion. If the bandwidth of the pulse encompasses a substantial portion of a resonance structure, it becomes necessary to retain a large number of terms to describe accurately the phase delay. Moreover, as was 
pointed out in Ref. [4], if the bandwidth exceeds the modulus of the difference between the carrier frequency and the nearest pole in the refractive index, the series fails to converge. These difficulties are no doubt the reasons for the expressions of caution when interpreting group velocity. In this Letter, we examine the role of group velocity without resorting to Eq. (1). We also distinguish between propagation effects and those of pulse reshaping due to spectral modification (absorption or amplification).

When considering the flow of energy of a light pulse, an essential fact is that the field is solely responsible for energy transport. This is true even though the total energy of a pulse is composed of energy in the field as well as in the medium. In the present work, we are interested in pulse arrival time (to a detector, say) as opposed to pulse position (spread throughout a medium) [8]. Thus, the energy density is not directly relevant, but only its associated flux. Also, the energy density in the medium is not easily assessed experimentally whereas the Poynting flux can be measured directly. Therefore, we will not use the energy transport velocity [3], defined traditionally as the ratio of the Poynting flux to the energy density, which renders luminal $[9,10]$ or superluminal [7] results dependent on the choice for energy density.

For the reasons given, the definition of the arrival time of pulse energy to a point need only involve the Poynting vector. As a direct consequence of Maxwell's equations, the Poynting flux is $\overrightarrow{\mathrm{S}}(\overrightarrow{\mathrm{r}}, t)=\overrightarrow{\mathrm{E}}(\overrightarrow{\mathrm{r}}, t) \times \overrightarrow{\mathrm{H}}(\overrightarrow{\mathrm{r}}, t)$, where in our notation $\overrightarrow{\mathrm{E}}$ and $\overrightarrow{\mathrm{H}}$ are the real electric and magnetic fields, respectively. To deal with arbitrary broad band pulses, the arrival time should avoid presupposing a specific pulse shape since the pulse may evolve in complicated ways during propagation. For example, the pulse peak or the midpoint on the rising edge are poor indicators of arrival time if the pulse contains multiple peaks or a long and nonuniform rise time. Instead, we will use a time expectation integral over the incoming Poynting flux to define the pulse arrival time. This approach, proposed for use with light pulses in 1970 by Smith [11], is similar to the expectation integral used in quantum mechanics, and indeed it has been used to describe the expected arrival time of single-photon wave packets [12]. This arrival time definition is also suitable for energetic pulses where the light is treated classically. Experimental techniques developed in recent years enable the accurate and routine temporal characterization of even very short light pulses (a few femtoseconds) [13], thus permitting an expectation integral to be performed.

The expectation integral (or time "center of mass") describing the arrival time of the pulse is given by

$$
\langle t\rangle_{\overrightarrow{\mathrm{r}}} \equiv \hat{u} \cdot \int_{-\infty}^{\infty} t \overrightarrow{\mathrm{S}}(\overrightarrow{\mathrm{r}}, t) d t / \hat{u} \cdot \int_{-\infty}^{\infty} \overrightarrow{\mathrm{S}}(\overrightarrow{\mathrm{r}}, t) d t .
$$

The unit vector $\hat{u}$ refers to the direction in which the energy flow is detected (normal to a detector surface). It has importance for angularly dispersive systems such as grating pairs where the result of the integral in the numerator is not necessarily parallel to that in the denominator. For clarity of exposition, we presently do not consider the angularly dispersive case but treat it elsewhere [14].

It is enlightening to consider the form of Eq. (2) in the frequency domain, where the fields are expressed as superpositions of pure frequency components:

$$
\begin{aligned}
\overrightarrow{\mathrm{E}}(\overrightarrow{\mathrm{r}}, t) & =\frac{1}{\sqrt{2 \pi}} \int_{-\infty}^{\infty} \overrightarrow{\mathrm{E}}(\overrightarrow{\mathrm{r}}, \omega) e^{-i \omega t} d \omega, \\
\overrightarrow{\mathrm{H}}(\overrightarrow{\mathrm{r}}, t) & =\frac{1}{\sqrt{2 \pi}} \int_{-\infty}^{\infty} \overrightarrow{\mathrm{H}}(\overrightarrow{\mathrm{r}}, \omega) e^{-i \omega t} d \omega .
\end{aligned}
$$

The expected arrival time of the pulse $\langle t\rangle_{\overrightarrow{\mathrm{r}}}$ can be computed directly from the spectra of the fields, $\overrightarrow{\mathrm{E}}(\overrightarrow{\mathrm{r}}, \omega)$ and $\overrightarrow{\mathrm{H}}(\overrightarrow{\mathrm{r}}, \omega)$, which contain the necessary phase information. The results presented in this Letter are obtained from the following form of Eq. (2):

$$
\langle t\rangle_{\overrightarrow{\mathrm{r}}}=T[\overrightarrow{\mathrm{E}}(\overrightarrow{\mathrm{r}}, \omega)],
$$

where $T[\overrightarrow{\mathrm{E}}(\overrightarrow{\mathrm{r}}, \omega)] \equiv-i \frac{\hat{u} \cdot \int_{-\infty}^{\infty} \frac{\partial \overrightarrow{\mathrm{E}}(\overrightarrow{\mathrm{r}}, \omega)}{\partial \omega} \times \overrightarrow{\mathrm{H}}^{*}(\overrightarrow{\mathrm{r}}, \omega) d \omega}{\hat{u} \cdot \int_{-\infty}^{\infty} \overrightarrow{\mathrm{S}}(\overrightarrow{\mathrm{r}}, \omega) d \omega}$.

The denominators in Eqs. (2) and (4) are equivalent in accordance with Parseval's theorem, where the Poynting vector in the frequency domain is defined by $\vec{S}(\vec{r}, \omega) \equiv$ $\overrightarrow{\mathrm{E}}(\overrightarrow{\mathrm{r}}, \omega) \times \overrightarrow{\mathrm{H}}^{*}(\overrightarrow{\mathrm{r}}, \omega)$.

The individual frequency components obey the Helmholtz equation $\nabla^{2} \overrightarrow{\mathrm{E}}(\overrightarrow{\mathrm{r}}, \omega)+\omega^{2} \varepsilon(\omega) \mu_{0} \overrightarrow{\mathrm{E}}(\overrightarrow{\mathrm{r}}, \omega)=0$. The solution to this equation in a uniform medium is given by $\overrightarrow{\mathrm{E}}(\overrightarrow{\mathrm{r}}, \omega)=\overrightarrow{\mathrm{E}}\left(\overrightarrow{\mathrm{r}}_{0}, \omega\right) \exp \{\overrightarrow{\mathrm{k}} \cdot \Delta \overrightarrow{\mathrm{r}}\}$, where the position is $\overrightarrow{\mathrm{r}}=\overrightarrow{\mathrm{r}}_{0}+\Delta \overrightarrow{\mathrm{r}}$, and where the wave number satisfies $k^{2}=\omega^{2} \varepsilon(\omega) \mu_{0}$. (The complex refractive index is the combination $k c / \omega$.) For clarity, we assume a single wave vector $\vec{k}(\omega)$ for each $\omega$. The spectrum of the magnetic field is tied to that of the electric field via the relation $\overrightarrow{\mathrm{H}}(\overrightarrow{\mathrm{r}}, \omega)=\overrightarrow{\mathrm{k}} \times \overrightarrow{\mathrm{E}}(\overrightarrow{\mathrm{r}}, \omega) / \omega \mu_{0}$. A knowledge of $\overrightarrow{\mathrm{k}}(\omega)$ and $\overrightarrow{\mathrm{E}}\left(\overrightarrow{\mathrm{r}}_{0}, \omega\right)$ for all $\omega$ is enough to specify the fields and the Poynting vector at all points $\vec{r}$ in the uniform medium.

The main point of this Letter is to introduce the following theorem and to explain its significance. By injecting the solution to the Helmholtz equation into Eq. (4), and after some manipulation, it can be shown that the time delay $\Delta t \equiv\langle t\rangle_{\overrightarrow{\mathrm{r}}}-\langle t\rangle_{\overrightarrow{\mathrm{r}}_{0}}$ between the passage of the pulse at $\overrightarrow{\mathrm{r}}_{0}$ and $\overrightarrow{\mathrm{r}}=\overrightarrow{\mathrm{r}}_{0}+\Delta \overrightarrow{\mathrm{r}}$ is given by

$$
\Delta t=G_{\overrightarrow{\mathrm{r}}}+R_{\overrightarrow{\mathrm{r}}_{0}} .
$$

The two terms in Eq. (5) have clear physical interpretations and are described below.

The first term which we will call the net group delay is a spectral average of the group delay of individual frequencies:

$$
\begin{aligned}
G_{\overrightarrow{\mathrm{r}}} & \equiv\left\langle\frac{\partial \operatorname{Re} \overrightarrow{\mathrm{k}}}{\partial \omega} \cdot \Delta \overrightarrow{\mathrm{r}}\right\rangle_{\overrightarrow{\mathrm{r}}} \\
& =\frac{\hat{u} \cdot \int_{-\infty}^{\infty} \overrightarrow{\mathrm{S}}(\overrightarrow{\mathrm{r}}, \omega)\left[\frac{\partial \operatorname{Re} \overrightarrow{\mathrm{k}}}{\partial \omega} \cdot \Delta \overrightarrow{\mathrm{r}}\right] d \omega}{\hat{u} \cdot \int_{-\infty}^{\infty} \overrightarrow{\mathrm{S}}(\overrightarrow{\mathrm{r}}, \omega) d \omega} .
\end{aligned}
$$


Note the close resemblance to Eq. (2). Equation (6) is the same center-of-mass or expectation integral, but executed in the frequency domain on $\partial \mathrm{Re} \overrightarrow{\mathrm{k}} \cdot \Delta \overrightarrow{\mathrm{r}} / \partial \omega$, or group delay. Thus, the inverse of group velocity at every frequency present in the pulse influences the result. This expectation integral is performed over that spectrum which survives the propagation to the final position $\vec{r}$. The net group delay depends only on the spectral content of the pulse, independent of its temporal organization [i.e., the phases of $\overrightarrow{\mathrm{E}}(\overrightarrow{\mathrm{r}}, \omega)$ and $\overrightarrow{\mathrm{H}}(\overrightarrow{\mathrm{r}}, \omega)$ do not contribute]. Only the real part of the refractive index plays a direct role in Eq. (6).

The second term in Eq. (5) represents a delay which arises solely from a reshaping of the spectrum through absorption (or amplification) and is given by

$$
R_{\overrightarrow{\mathrm{r}}_{0}} \equiv T\left[e^{-\operatorname{Im} \overrightarrow{\mathrm{k}} \cdot \Delta \overrightarrow{\mathrm{r}}} \overrightarrow{\mathrm{E}}\left(\overrightarrow{\mathrm{r}}_{0}, \omega\right)\right]-T\left[\overrightarrow{\mathrm{E}}\left(\overrightarrow{\mathrm{r}}_{0}, \omega\right)\right] .
$$

This reshaping delay is evaluated at $\vec{r}_{0}$, before propagation takes place. The reshaping delay is the difference between the pulse arrival time at the initial point $\vec{r}_{0}$ evaluated without and with the spectral amplitude that is lost during propagation. Both terms in Eq. (7) utilize the phase of the fields at $\vec{r}_{0}$. In contrast to the net group delay, the reshaping delay is sensitive to how the pulse is organized. The reshaping delay is zero if the spectrum of the pulse is unaltered during propagation. This occurs if the imaginary part of the refractive index is negligible. It also occurs in the narrow band limit even if pulses experience strong absorption (or amplification), as will be discussed later.

Before illustrating the use of Eq. (5), it is interesting to note that the ordering for the evaluation of the net group delay and reshaping delay can be reversed. In other words, the subscripts $\vec{r}$ and $\vec{r}_{0}$ in Eq. (5) can be interchanged. If the group delay is computed with the initial spectrum instead of the spectrum that survives the propagation, then the reshaping delay must be evaluated at the end of propagation without and with the lost spectral amplitude (i.e., $\left.R_{\overrightarrow{\mathrm{r}}}=T[\overrightarrow{\mathrm{E}}(\overrightarrow{\mathrm{r}}, \omega)]-T[\exp \{\operatorname{Im} \overrightarrow{\mathrm{k}} \cdot \Delta \overrightarrow{\mathrm{r}}\} \overrightarrow{\mathrm{E}}(\overrightarrow{\mathrm{r}}, \omega)]\right)$. This can dramatically alter the group and reshaping delays taken individually. Nevertheless, their sum $\Delta t$ is unaffected by the ordering. Either ordering converges to the same accumulated group and reshaping delays when the displacement $\Delta \overrightarrow{\mathrm{r}}$ is subdivided into many intervals.

Equation (5) can be used to examine the situations of the type elucidated in Refs. [4-7]. To do this, we employ the Lorentz model with a single resonance at $\omega_{0}$ and a damping frequency $\gamma$. In accordance with the model, the square of the refractive index is $1+f \omega_{p}^{2} /\left(\omega_{0}^{2}-\omega^{2}-i \gamma \omega\right)$, where $\omega_{p}$ is the plasma frequency and $f$ is the oscillator strength. Figure 1(a) depicts the real and imaginary parts of the index where we have chosen the model parameters to be $\omega_{0}=100 \gamma$ and $f \omega_{p}^{2}=100 \gamma^{2}$.

The electric field at $\overrightarrow{\mathrm{r}}_{0}$ is chosen to be Gaussian, $\overrightarrow{\mathrm{E}}\left(\overrightarrow{\mathrm{r}}_{0}, t\right)=\hat{x} E_{0} \exp \left\{-t^{2} / \tau^{2}\right\} \cos (\bar{\omega} t)$, with initial durations $\tau_{1}=10 / \gamma$ (narrow band) and $\tau_{2}=1 / \gamma$ (broad band). Figures 1(b) and 1(c) depict the spectral intensity
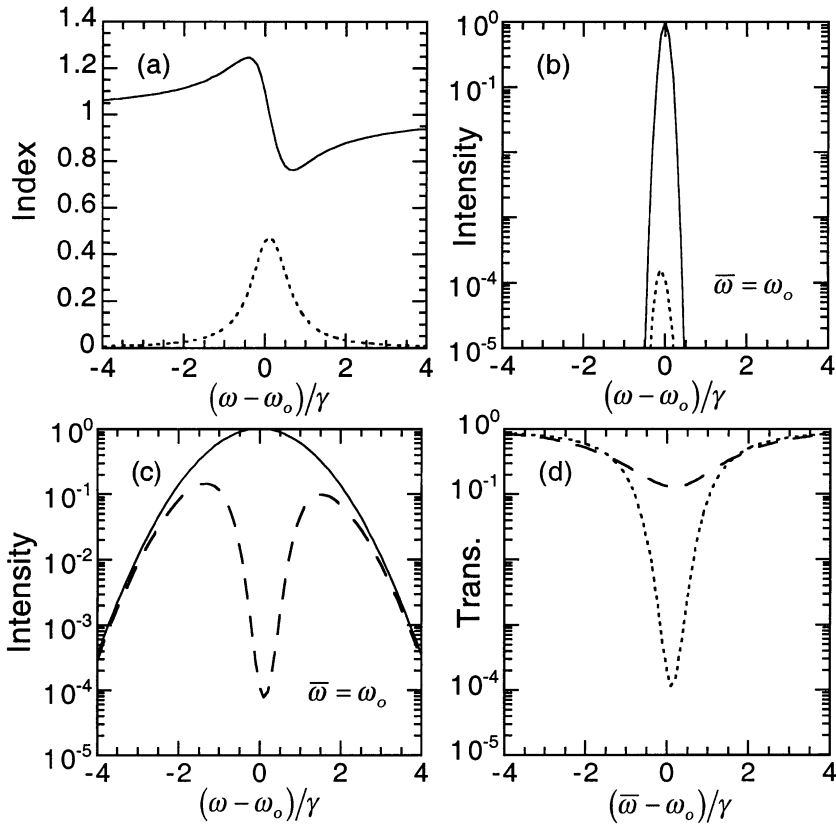

FIG. 1. (a) Real (solid line) and imaginary (dotted line) parts of the refractive index. (b) Initial spectrum (solid line) and spectrum after propagation (dotted line) of the narrow band pulse with $\bar{\omega}=\omega_{0}$. (c) Initial spectrum (solid line) and spectrum after propagation (dashed line) of the broad band pulse with $\bar{\omega}=\omega_{0}$. (d) Transmission of the narrow band pulse (dotted line) and the broad band pulse (dashed line) as a function of $\bar{\omega}$.

of the two pulses before propagation in the medium (solid lines) with each spectrum centered on the resonance (i.e., $\left.\bar{\omega}=\omega_{0}\right)$. The dotted line and the dashed line show the two spectra after propagation through a displacement $\Delta \overrightarrow{\mathrm{r}}=\hat{z} c /(10 \gamma)$, with $\overrightarrow{\mathrm{k}}=k \hat{z}$. Figure $1(\mathrm{~d})$ shows the fraction of pulse energy which survives the propagation as the pulse's central frequency $\bar{\omega}$ is varied. The broad band pulse (dashed line) is less attenuated relative to the narrow band pulse (dotted line) since it is wider than the absorbing resonance.

Figure 2 shows the delay between the pulse arrival times at $\vec{r}_{0}$ and $\vec{r}=\vec{r}_{0}+\Delta \vec{r}$ as the pulse's central frequency $\bar{\omega}$ is varied. The identical solid curves in Figs. 2(a) and 2(b) give the total delay $\Delta t$ experienced by the narrow band pulse in traversing the displacement. The dashed line in Fig. 2(a) represents the net group delay $G_{\overrightarrow{\mathrm{r}}}$ evaluated over the final spectrum, while the dotted line shows the reshaping delay $R_{\overrightarrow{\mathrm{r}}_{0}}$ evaluated at the initial position. In Fig. 2(b) the ordering is reversed: The net group delay $G_{\overrightarrow{\mathrm{r}}_{0}}$ is evaluated over the initial spectrum while the reshaping delay $R_{\overrightarrow{\mathrm{r}}}$ is evaluated at the end of propagation.

When the spectrum is narrow compared to nearby features in the resonance, the reshaping delay tends to zero whether evaluated at the beginning or at the end of propagation. In this case, the total delay is dominated by the net group delay and reduces to $\lim _{\tau \rightarrow \infty} \Delta t=G=$ $\Delta r \partial \operatorname{Re} k /\left.\partial \omega\right|_{\bar{\omega}}$. This recovers the narrow band result explored in Refs. [4-7] where the pulse travels at the group 

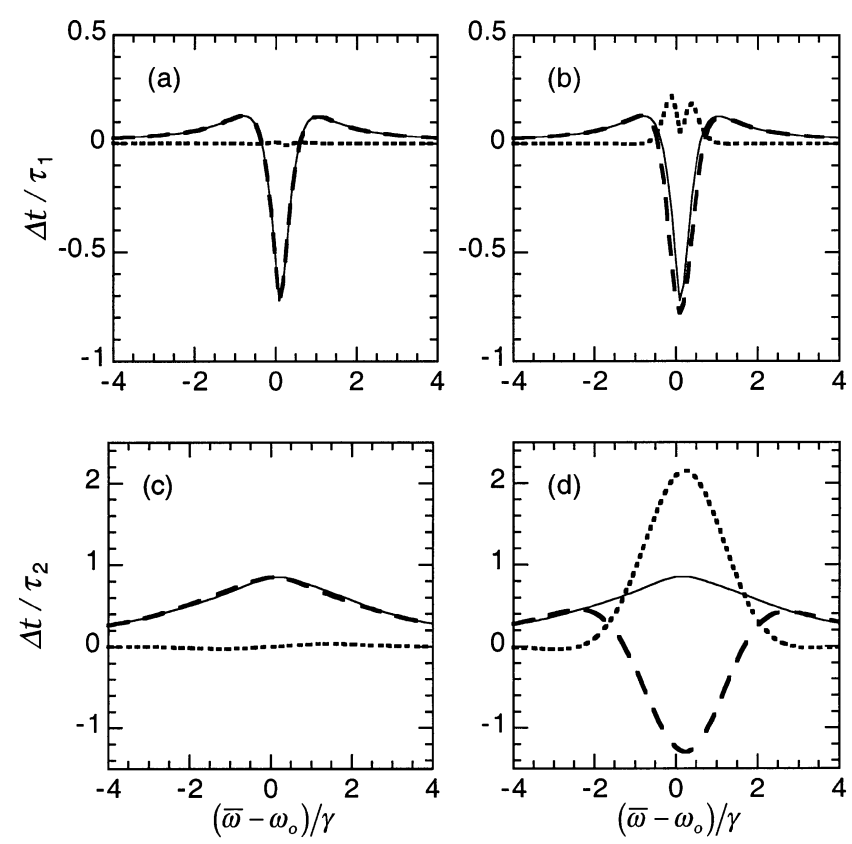

FIG. 2. (a) Group delay (dashed line), reshaping delay (dotted line), and total delay (solid line) as a function of $\bar{\omega}$ for the narrow band pulse. (b) Same as (a) but with reshaping computed at the end of propagation. Frames (c) and (d) repeat (a) and (b) for the case of the broad band pulse.

velocity evaluated at $\bar{\omega}$. Figures 2(a) and 2(b) are not far from this limit, exhibiting superluminal and negative propagation speeds (i.e., the Garrett and McCumber effect) [4]. Nevertheless, the temporal advance is similar to the duration of the pulse, and the emerging light is well within the original pulse envelope propagated forward at speed $c$. As described in Ref. [7], somewhat larger pulse advances relative to pulse duration are possible when propagation takes place below a resonance in an amplifying medium (negative $f$ ), and we have used Eq. (5) to observe this effect.

Figures 2(c) and 2(d) repeat Figs. 2(a) and 2(b) for the broad band pulse. This pulse which is initially more localized in time does not propagate with superluminal speed. The net group delay can go negative if computed using the initial spectrum as in Fig. 2(d), but the corresponding reshaping delay (evaluated at the end) causes the overall result to remain luminal as the pulse experiences a strong chirp during propagation. If the delays were to be evaluated by summing over contributions from many subdivisions of the displacement $\Delta \overrightarrow{\mathrm{r}}$, then the resulting net group and reshaping delays would lie between those in Figs. 2(c) and 2(d). Nevertheless, we have opted to present our formalism in terms of the difference in arrival times at the end points, such as might be measured in an experiment, rather than in terms of an analysis over the continuum of points lying in between. It is possible to define a velocity [11] at every point along the displacement $\Delta \overrightarrow{\mathrm{r}}$, the inverse of which being the gradient of Eq. (2), but it should be kept in mind that this velocity varies continually to the extent that the reshaping delay is present.

The extreme broad band limit of Eq. (5) is also instructive. When the duration of the pulse goes to zero, becoming a delta function with a sharply defined position, the spectral content of the pulse extends uniformly over all frequencies. By comparison, any resonance structure encompasses a finite bandwidth, and the reshaping delay tends to zero. Thus, as in the narrow band limit, the total delay is dominated by the net group delay which reduces to $\lim _{\tau \rightarrow 0} \Delta t=G=\Delta r / c$, assuming a physically realistic refractive index (approaching unity at high frequency). This demonstrates the well known fact that the velocity of a sharply defined signal is exactly $c$.

In summary, without relying on approximations we have found a context where group velocity is always meaningful. The real part of the refractive index gives rise to a net group delay which is the spectral superposition of group delay at each frequency. An accompanying reshaping delay arises from spectral modification through the imaginary part of the refractive index. The results are based on the specific center-of-mass definition of pulse arrival time, motivated in part because it may handle arbitrary pulse shapes. This choice is also justified by the fact that it leads to Eq. (5) with its clear interpretation. The well known Sommerfeld result [3] of luminality for pulses of definite support is consistent with and even implied by the present context.

*Department of Mathematics.

[1] M. Born and E. Wolf, Principles of Optics (Pergamon, Oxford, 1980), 6th ed., pp. 20, 23.

[2] J.D. Jackson, Classical Electrodynamics (Wiley, New York, 1998), 3rd ed., pp. 323, 325.

[3] L. Brillouin, Wave Propagation and Group Velocity (Academic Press, New York, 1960).

[4] C. G. B. Garrett and D.E. McCumber, Phys. Rev. A 1, 305-313 (1970).

[5] S. Chu and S. Wong, Phys. Rev. Lett. 48, 738-741 (1982).

[6] R. Y. Chiao, Phys. Rev. A 48, R34-R37 (1993).

[7] E. L. Bolda, J. C. Garrison, and R. Y. Chiao, Phys. Rev. A 49, 2938-2947 (1994).

[8] S. A. Glasgow and J. Peatross (to be published).

[9] G. Diener, Phys. Lett. A 235, 118-124 (1997).

[10] R. Loudon, J. Phys. A 3, 233-245 (1970).

[11] R. L. Smith, Am. J. Phys. 38, 978-983 (1970).

[12] W. Yun-ping and Z. Dian-lin, Phys. Rev. A 52, 2597-2600 (1995).

[13] R. Trebino, K.W. DeLong, D. N. Fittinghoff, J. N. Sweetser, M. A. Krumbugel, and B. A. Richman, Rev. Sci. Instrum. 68, 3277-3295 (1997).

[14] M. Ware, W. E. Dibble, S. A. Glasgow, and J. Peatross (to be published). 